\title{
A Fuzzy Reliability Analysis and Simulation Method of Hydraulic System
}

\author{
LI Feng, YUAN Zhi-kai ${ }^{\text {a, }}$, HE Zhen-xin \\ Xi'an High Technology Research Institute,Xi'an,China \\ e-mail:sdaqyzk@163.com
}

Keywords: Hydraulic system, Fault tree, Monte Carlo simulation.

\begin{abstract}
A Monte Carlo reliability simulation method based on T-S fuzzy fault tree model is proposed to solve the problem of hydraulic system reliability modeling and simulation. Firstly, a reliability analysis model of hydraulic system based on T-S fuzzy fault tree model is established. Secondly, based on the reliability model, the simulation of the system reliability is realized by the fusion Monte Carlo numerical simulation method. Finally, the effectiveness of the method is verified by the typical hydraulic circuit. In the case of simulation 2000 times, compared with the theoretical calculation the error of Simulation results is on the order of $10^{-2}$.
\end{abstract}

\section{Introduction}

With the development of technology, hydraulic technology has been widely used in construction machinery and mine machinery. In some cases, the reliability of the hydraulic system is directly related to the life safety of the property. The use of reliability analysis can effectively improve the efficiency of hydraulic system design and maintenance and shorten the reliability of design time ${ }^{[1,2]}$.

Traditional fault tree analysis method is simple and logical, which is an effective method for qualitative analysis of system reliability ${ }^{[3,4]}$. However, there are three main problems such as lack of fault probability acquisition, difficult to determine the event contact and can't describe the fault degree, so the system reliability quantitative calculation is often greater deviation. In order to solve the problem of quantitative calculation of traditional fault tree, a reliability analysis method of T-S fuzzy fault tree is proposed ${ }^{[5]}$. This method solves the problem of failure probability uncertainty by describing the probability of failure with fuzzy number. By constructing the T-S fuzzy gate to describe the inter-event link, the problem of event contact uncertainty is solved. Through the introduction of expert experience, the problem of lacking fault probability access is made up.

Monte Carlo simulation is a statistical experiment based on random variables or stochastic simulation method, which can deal with complex system reliability analysis problems with different life distribution and reliability model $s^{[6,7]}$. It can also reflect the randomness of system faults, and is widely used in the field of reliability simulation.

In this paper, the Monte Carlo simulation method is combined with the reliability analysis method of T-S fuzzy fault tree, and a fuzzy reliability analysis and simulation method of hydraulic system is proposed to assist the hydraulic system reliability design and shorten the reliability design calculation time.

\section{T-S Fuzzy Fault Tree Analysis Method}

\subsection{Build membership function}

Usually we describe the fault as "no fault", "moderate fault ", "serious fault ", the exact value can't describe the degree of failure, so use $[0,1]$ on the fuzzy number to describe ${ }^{[8]}$. For the convenience of calculation and description without loss of generality, select the symmetrical trapezoidal membership function, the expression is as follows: 


$$
\mu(x)= \begin{cases}0 & x_{0}+s_{r}+m_{r} \leq x \leq x_{0}-s_{l}-m_{l} \\ \frac{x-\left(x_{0}-s_{l}-m_{l}\right)}{m_{l}} & x_{0}-s_{l}-m_{l} \leq x \leq x_{0}-s_{l} \\ 1 & x_{0}-s_{l} \leq x \leq x_{0}+s_{r} \\ \frac{x_{0}+s_{r}+m_{r}-x}{m_{r}} & x_{0}+s_{r} \leq x \leq x_{0}+s_{r}+m_{r}\end{cases}
$$

The membership function graphs of the three fault states are given as $0,0.5,1$ respectively, as the support center of the three fault state fuzzy numbers, and the membership function is chosen as follows:

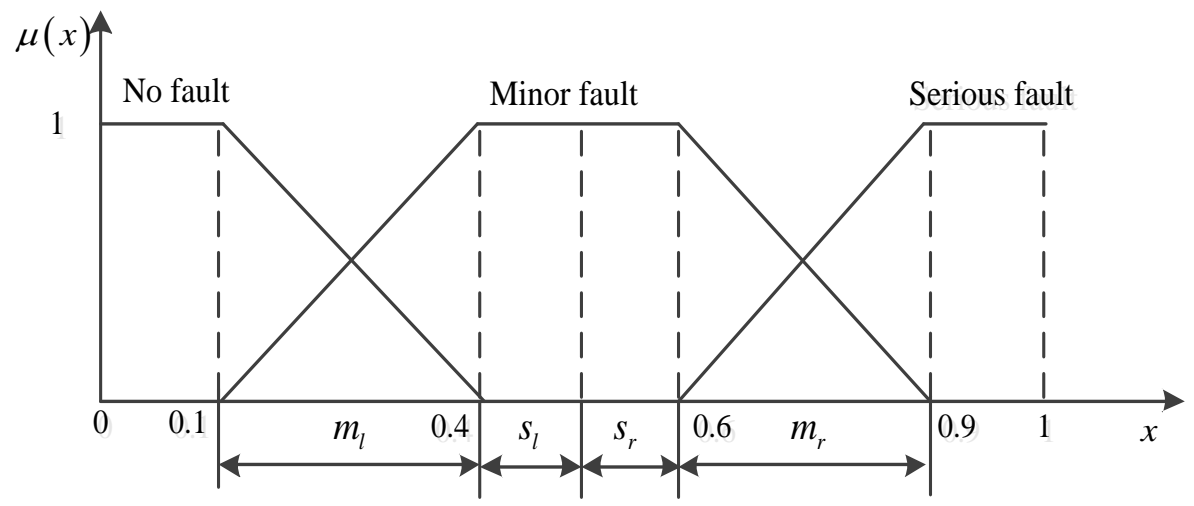

Fig.1 Membership Function Graphs of Three Fault State

\subsection{T-S fuzzy tree algorithm}

Let the basic event be $\left\{x_{1}, x_{2}, \cdots, x_{n}\right\}$ and the upper event be $Y$, and they are connected by T-S fuzzy gate. The degree of failure is described by fuzzy number $\left(x_{1}^{1}, x_{1}^{2}, \cdots, x_{1}^{k_{1}}\right),\left(x_{2}^{1}, x_{2}^{2}, \cdots, x_{2}^{k_{1}}\right)$, $\cdots, \quad\left(x_{n}^{1}, x_{n}^{2}, \cdots, x_{n}^{k_{1}}\right)$ and $\left(y^{1}, y^{2}, \cdots, y^{n}\right)$ respectively.

The rules $l$ of the T-S fuzzy gate can be defined as follows:

$$
\left\{p^{l}\left(\mathrm{y}^{1}\right), p^{l}\left(\mathrm{y}^{2}\right), \cdots, p^{l}\left(\mathrm{y}^{n}\right) \mid x_{1}=x_{1}^{i_{1}}, \cdots, x_{n}=x_{n}^{i_{n}}\right\}
$$

Where $i_{1}=1,2, \cdots, k_{1}, \quad i_{2}=1,2, \cdots, k_{2}, \cdots$ The rule $l$ indicates that when the basic event $\left\{x_{1}, x_{2}, \cdots, x_{n}\right\}$ is in a fault state $\left\{x_{1}=x_{1}^{i_{1}}, x_{2}=x_{2}^{i_{2}}, \cdots, x_{n}=x_{n}^{i_{n}}\right\}$, and the superior event is in each fault state $\left\{Y=\mathrm{y}^{k}\right\}$, the probability is $p^{l}\left(\mathrm{y}^{k}\right)$. The total number of rules is: $m=\prod_{i=1}^{n} k_{i}$.

When the basic event is $\left\{x_{1}=x_{1}^{i_{1}}, x_{2}=x_{2}^{i_{2}}, \cdots, x_{n}=x_{n}^{i_{n}}\right\}$, the rule execution probability is

$$
p_{0}^{l}=p\left(x_{1}^{i_{1}}\right) p\left(x_{2}^{i_{2}}\right) \cdots p\left(x_{n}^{i_{n}}\right)
$$

So the fuzzy possibility of the superior event is:

$$
\left\{\begin{array}{c}
p\left(\mathrm{y}^{1}\right)=\sum_{l=1}^{m} p_{0}^{l} p^{l}\left(\mathrm{y}^{1}\right) \\
p\left(\mathrm{y}^{2}\right)=\sum_{l=1}^{m} p_{0}^{l} p^{l}\left(\mathrm{y}^{2}\right) \\
\vdots \\
p\left(\mathrm{y}^{n}\right)=\sum_{l=1}^{m} p_{0}^{l} p^{l}\left(\mathrm{y}^{n}\right)
\end{array}\right.
$$




\section{Monte Carlo Numerical Simulation}

Through the T-S fuzzy fault tree analysis method, the failure rate of the top event can be obtained by the failure rate of the underlying event, and the Monte Carlo numerical simulation method is used to simulate the system reliability.

\subsection{The basic principle of Monte Carlo simulation}

Assume that the structural limit state equation is $g_{m}(\cdot)=0, m=1,2, \cdots, n_{f}$, Where $n_{f}$ is the number of the limit state equation. If only consider the structure of the two states, $g_{m}(\cdot)>0$ is a safe state, $g_{m}(\cdot)<0$ is a failure state, known as the structural reliability of the two-state model ${ }^{[9]}$. The corresponding failure probability can be called the classical failure probability, and because only considering the randomness of this kind of uncertainty, it can be called the random failure probability, the exact solution of the failure probability is

$$
P=\int_{D_{f}} \cdots \int f(\vec{x}) d \vec{x}
$$

Where $f(\bar{x})$ is the joint probability density function of the basic random variable vector $\bar{x}$, and $D_{f}$ is the system failure domain constituted by $g_{m}(\cdot)<0$.

The basic idea of the direct Monte Carlo method is to use the joint probability density function $f(\vec{x})$ of the basic random variable to be sampled. The ratio of the number $N_{f}$ of the sample points falling into the failure domain $D_{f}$ to the number $N$ of the total sample points is given as the formula (1) .The unbiased estimate of the failure probability $\bar{P}$ defined in Eq.(5), which is

$$
\bar{P}=\frac{N_{f}}{N}=\frac{1}{N} \sum_{i=1}^{N} I\left[D_{f}: \mathrm{g}_{m}\left(v^{(i)}\right) \leq 0, m=1,2, \cdots, n_{f}\right]
$$

Where $v^{(i)}$ is the $i$ th sample of the basic random variable vector, $T[\cdot]$ is the state indicating function. When the sample point falls within the invalid domain, $T[\cdot]=1$; otherwise $T[\cdot]=0$.

\subsection{Monte Carlo algorithm steps}

Based on the analysis of the Monte Carlo numerical simulation principle, we can summarize the Monte Carlo simulation algorithm steps as follows:

Step1 Produce independent random numbers uniformly distributed over the interval [0,1], where $i=1,2, \cdots, N, j=1,2, \cdots, n . N$ is the number of sample groups sampled, $n$ is the number of basic variables;

Step2 The above random samples are converted into $N$ sets of random vector samples that correspond to the cumulative distribution function $F_{j}$ of the basic random variable $x_{j}$, where $x_{i j}=F_{j}^{-1}\left(r_{i j}\right)$.

Step3 Substituting the sample $\vec{x}_{i}$ of the random vector into the limit state equation and accumulating it according to the state indication function $T_{j}[\cdot]$;

Step4 Solve the failure probability estimate $P$ by Eq. (6).

The Monte Carlo method is not limited to the scope of application and has nothing to do with the number of variables, as long as the limit state equation can be known. However, for the small probability event common to engineering, it is necessary to make $\mathrm{N}$ very large to converge ${ }^{[10]}$. Therefore, it is generally necessary to use a large number of points to improve the accuracy and reduce the variance. 


\section{Case Analysis}

Truck crane is a common construction machinery. Because the tires of the truck crane are elastomers and its support force is limited, so the lifting operation must be carried out before the leg to load ${ }^{[11]}$. When the truck crane is running, the legs need to be stowed. In the course of work, the frequent system failure of truck crane leg circuit is can't lay out the legs or decentralized legs but can't hold up the body. Analysis of the causes of system failure, the main reasons are: 1. Hydraulic pump failure, 2. Oil filter failure, 3. Valve failure, 4. Two-way hydraulic lock failure, 5. Relief valve failure, 6. Hydraulic cylinder failure . The establishment of T-S fault tree as shown in Fig.2.

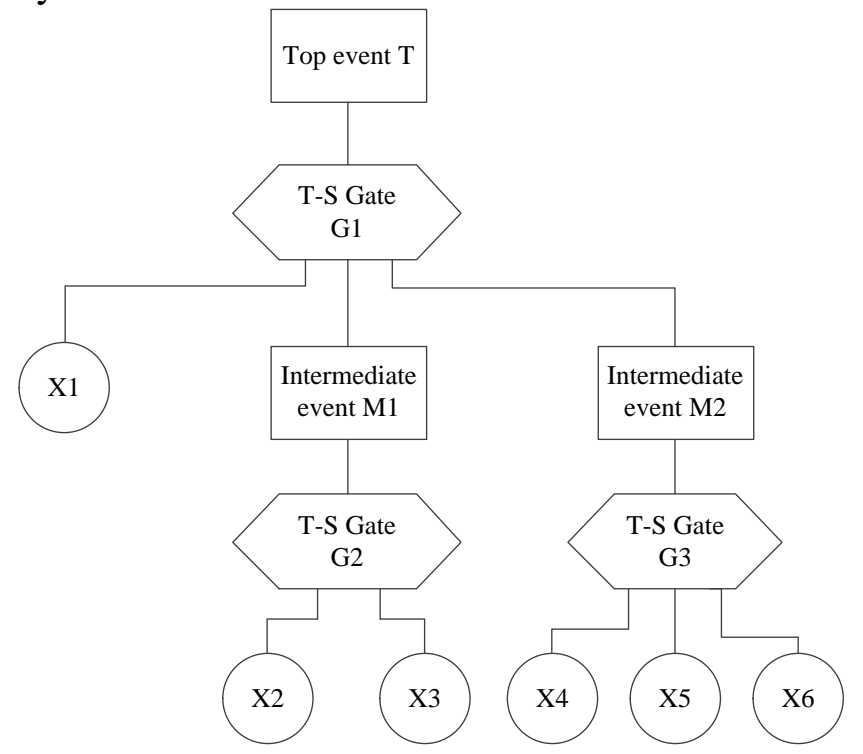

Fig.2 Outrigger hydraulic circuit T-S fuzzy fault tree

$\mathrm{T}$ - The legs can't support the body $\mathrm{M}_{1}$-Fuel supply system failure $\mathrm{M}_{2}$-Control loop failure $\mathrm{X}_{1}$ Hydraulic cylinder failure $\mathrm{X}_{2}$-Hydraulic pump failure $\mathrm{X}_{3}$-Oil filter failure $\mathrm{X}_{4}$-Reversing valve failure $\mathrm{X}_{5}-$ Two-way hydraulic lock failure $\mathrm{X}_{6}$-Relief valve failure

Through the 10 hydraulic failure and maintenance experts given the empirical data based on academic qualifications and business time weighted processing, the fault tree in the three T-S door were developed. Based on the empirical data given by 10 experts on hydraulic failure and maintenance, the T-S fuzzy gate rule was developed according to the academic and practice time weighting. According to the manual of hydraulic components, the basic failure rate of each hydraulic element is obtained, and the above failure rate data are brought into the established T-S fuzzy fault tree model to calculate the failure rate of each event ${ }^{[12]}$.

Tab.1 Event failure rate calculation result

\begin{tabular}{|c|c|c|}
\hline Event & $\begin{array}{c}\text { Moderate failure rate } \\
10^{-7} \mathrm{~h}^{-1}\end{array}$ & $\begin{array}{c}\text { Serious failure rate } \\
10^{-6} \mathrm{~h}^{-1}\end{array}$ \\
\hline $\begin{array}{c}\text { Fuel supply system } \\
\left.\text { failure ( } \mathrm{M}_{1}\right)\end{array}$ & 18.4 & 6.25 \\
\hline $\begin{array}{c}\text { Control loop failure } \\
\left(\mathrm{M}_{2}\right)\end{array}$ & 15.6 & 6.13 \\
\hline $\begin{array}{c}\text { The legs can't } \\
\text { support the body (T) }\end{array}$ & 24.2 & 78.32 \\
\hline
\end{tabular}

Monte Carlo simulation is used to predict the reliability of the hydraulic system over time. Set the simulation number at 2000 times, select Matlab random number generation command to generate random number, calculated by the system reliability curve with time as shown in Figure.3: 


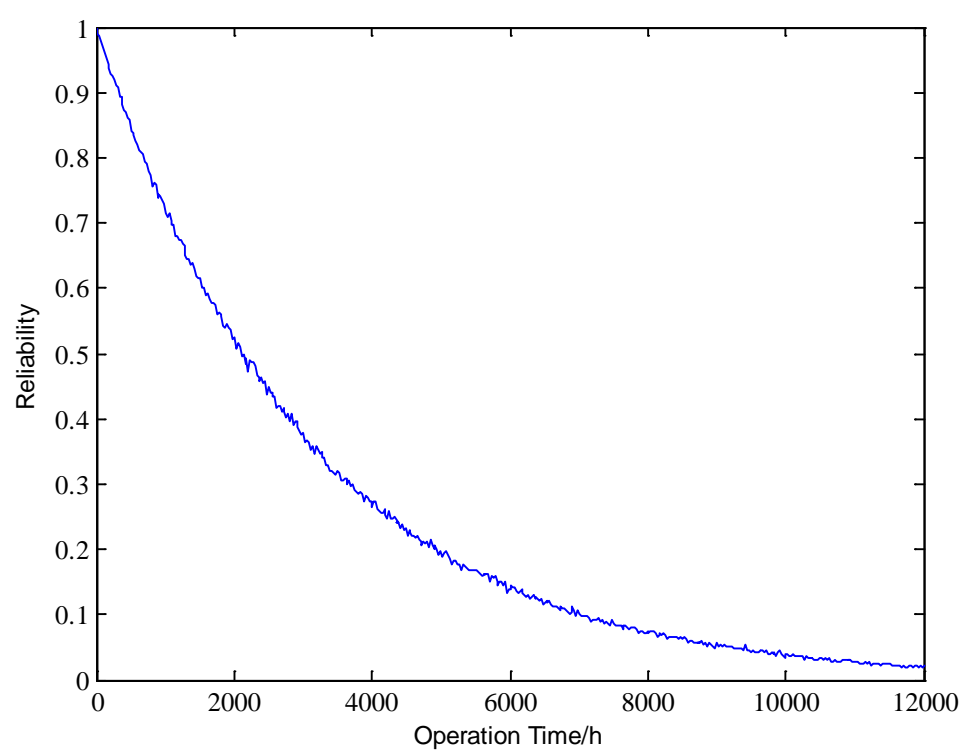

Fig.3 Simulation curve of system reliability change

From Figure. 3 can be seen in the car crane hydraulic circuit in the work of about 1500 h reliability down to 0.6 below the work of about $2400 \mathrm{~h}$ reliability down to 0.4 below.

In order to further analyze the accuracy of the simulation results, select the six time points in the working time, compare the reliability of the simulation with the reliability of the calculation by mathematical analysis. The results are shown in Table.2.

Tab.2 Comparison of reliability results

\begin{tabular}{|c|c|c|c|}
\hline Time/h & Analytical method & Simulation method & Error $/ \%$ \\
\hline 1000 & 0.7253 & 0.7280 & $0.37 \%$ \\
\hline 2000 & 0.5262 & 0.5237 & $0.47 \%$ \\
\hline 4000 & 0.2726 & 0.2737 & $0.41 \%$ \\
\hline 6000 & 0.1423 & 0.1453 & $2.10 \%$ \\
\hline 8000 & 0.0743 & 0.0745 & $0.26 \%$ \\
\hline 10000 & 0.0385 & 0.0360 & $6.47 \%$ \\
\hline
\end{tabular}

It can be seen from the comparison of the data in Table.2 that the simulation results of the Monte Carlo simulation method are about $10^{-2}$ when the number of simulations is 2000 times, which proves the validity of the Monte Carlo simulation method .

\section{Conclusion}

In this paper, the reliability of the truck crane leg loop is calculated by using the T-S fuzzy fault tree model, and the Monte Carlo simulation method is used to simulate the reliability of the system with time. The results show that:

1. The possibility of a moderate failure of the hydraulic circuit of the truck crane is at the same order of magnitude as the failure of the hydraulic components. The possibility of serious failure is higher than the possibility of serious failure of the hydraulic components two orders of magnitude.

2. The probability of failure of the hydraulic system of the truck crane is greater than that of the oil supply system, and the probability of failure of the oil supply system is greater than that of the control circuit.

3. The Monte Carlo simulation results show that the method is an effective method for hydraulic system reliability simulation analysis when the simulation number is 2000 times and the error is about $10^{-2}$.

The results show that the reliability analysis method based on the TS fuzzy fault tree model is an effective method for the reliability analysis of the hydraulic system, and the method can make up for the lack of fault history data by expert experience. , Which reduces the requirement of reliability analysis to fault history data, and is more adaptable than traditional fault tree analysis method. 


\section{References}

[1] YAO Cheng-yu ZHANG Ying-yi WANG Xu-feng, et al. Current Research and Developing Trends on Fault Tree Analysis of Hydraulic System [J]. Hydraulics Pneumatics \& Seals,2010,8:1923.

[2] ZHAO Jing-yi, YAO Cheng-yu. Progress of reliability research on hydraulic system[J]. Hydraulics Pneumatics\&Seals, 2006,3: 50-52.

[3] Yao Cheng-yu Zhao Jing-yi. Research on Fuzzy Fault Tree Analysis Method for Hydraulic System Based on T- S Model[J]. China Mechanical Engineering, 2009 20(16): 1913-1917.

[4] GAO Xing-pei. Fault Diagnosis of Hydraulic System in Automobile Crane Based on Binary Fault Tree and Case-Based Reasoning[J]. MACHINETOOL \& HYDRAULICS,2015, 43,(13): 198-200.

[5] SONG Hua, ZHANG Hong-yue, WANG Xing -ren. Fuzzy Fault Tree Analysis Based on T-S Model[J]. Control and Decision, 2005,20( 8): 854-859.

[6] HE Qing-gong, WANG Han-gong, CHEN Xiao-hu. Application of Fault Tree Analysis to Hydraulic System Fault Diagnosis in Automobile Crane[J].MACHINETOOL \& HYDRAULICS, 2008 , 36(2): 196-98.

[7] Seyed M L,Nahid R,Farinaz S.Utilisation of Fuzzy Fault Tree Analysis(FFTA) for quantified risk analysis of leakage in abandoned oil and natural-gas wells[J].Ocean Engineering,2015,108:729737.

[8] GAO Xin-gong. Reliability Analysis of Medium Speed Coal Mill Hydraulic System Based on Bayesian Network[J]. Master's Dissertation of Yan Shan University,2015:32-46.

[9] PAN H, YUN W. Fault tree analysis with fuzzy gates[J].Computers \& Industrial Engineering, 1997, 33(3) : 569-572.

[10] LAVASANI S M, ZENDEGANI A, CELIK M. Anextension to fuzzy fault tree analysis (FFTA) applicationin petrochemical process industry[J]. Process Safety and Environmental Protection, 2015, 93: 75-88.

[11] YAO Chengyu, CHEN Dongning, WANG Bin. Fuzzy reliability assessment method based on T-S fault tree and Bayesian network [J].Journal of Mechanical Engineering, 2014, 50(2): 193201.

[12] TANG Hongbin, WU Yunxin. Fault diagnosis of hydraulic system of concrete pump truck based on T-S fuzzy fault tree analysis[J]. Application Research of Computers， 2012(2): 561-564. 